\title{
Buckling Analysis of Functionally Graded Timoshenko Beams
}

\author{
S. Ayala, Bachelor degree, A. Vallejos, Bachelor degree, and R. Arciniega, Ph.D. \\ Universidad Peruana de Ciencias Aplicadas (UPC), Peru, u201514754@upc.edu.pe, u201514911@upc.edu.pe, \\ roman.arciniega@upc.edu.pe
}

\begin{abstract}
A finite element model based on the Timoshenko theory is developed to compute the critical buckling loading of functionally graded beams. The Trefftz criteria is used for the stability analysis considering both fundamental and incremental states. A finite element formulation is derived from the principle of virtual work. The Lagrange interpolation functions are used to approximate the field variables. Numerical results are validated with several benchmark problems found in literature.
\end{abstract}

Keywords - buckling analysis, functionally graded materials, stability, finite element method, Trefftz criterion

\section{INTRODUCTION}

It is important to understand the behavior and critical values of the structures under compressive loads to design more resistant and durable elements. Buckling analysis has been studied for many years since the pioneer work of Euler's critical buckling formula in the 18 th century $[1,2]$.

Functionally graded materials (FGM) are composites which properties vary continuously in one or more directions. It was firstly manufactured in Japan in 1987 with the objective to obtain heat-shielding for spacecraft [3]. Nowadays, it is applied in civil, naval and mechanical engineering projects due to their advantages in mechanical and thermal resistance. Also, FGM can avoid the critical stress concentrations, consequently, plastic deformations and cracking [4]. Generally, it is made of ceramic (high-temperature resistant) and metal (ductility and prevents fractures).

In literature, there are excellent contributions to studies of buckling elements with functionally graded materials. Reddy and Arciniega [5] analyzed the mechanical and thermal buckling of FGM plates. They developed a finite element model based on the third shear deformation theory (TSDT). The Trefftz criterion was employed for the buckling stability formulation. Li and Batra [6] calculated the critical buckling load for Timoshenko and Euler-Bernoulli's theories for three boundary conditions. They studied the buckling of FGM beams to compare both beam theories analytically and concluded that critical buckling load is easier to calculate by the Timoshenko theory. Kahya and Turan [7] obtained the buckling stability equation using Lagrange government equations, based on finite elements, to obtain the critical load for FGM Timoshenko beams. Huang and Li [8] performed stability for FGM beams with a circular cross-section. The authors considered shear deformation in their formulation, but not the shear correction factor for the calculation of the critical buckling load. Vo et al. [9] performed a finite element model based on the theory of refined shear deformation for functionally graded sandwich

Digital Object Identifier (DOI):

http://dx.doi.org/10.18687/LACCEI2020.1.1.512

ISBN: 978-958-52071-4-1 ISSN: 2414-6390 beams. They used the Reddy-Bickford beam theory to get the displacement field and Hamilton's theory for the variational formulation. The authors concluded that the effect of the geometrical parameters, boundary conditions and power-law index play a very important role in the analysis of vibration and buckling.

The aim of this study is to obtain the critical buckling load of FGM beams using a variational method called Trefftz criterion. The field displacement formulation is based on the Timoshenko beam theory for a finite element model. Also, the material properties of the FGM beam vary throughout the thickness considering a power-law index for three different boundary conditions simple supported, doubly clamped and clamped-free. Numerical results and the accuracy of the formulation have been compared and validated with other investigations based on literature.

\section{THEORETICAL FORMULATION}

\section{A. Kinematics and equilibrium equations}

In this study, we use the Timoshenko beam theory to represent the behavior of functionally graded beams, as shown in Fig. 1.

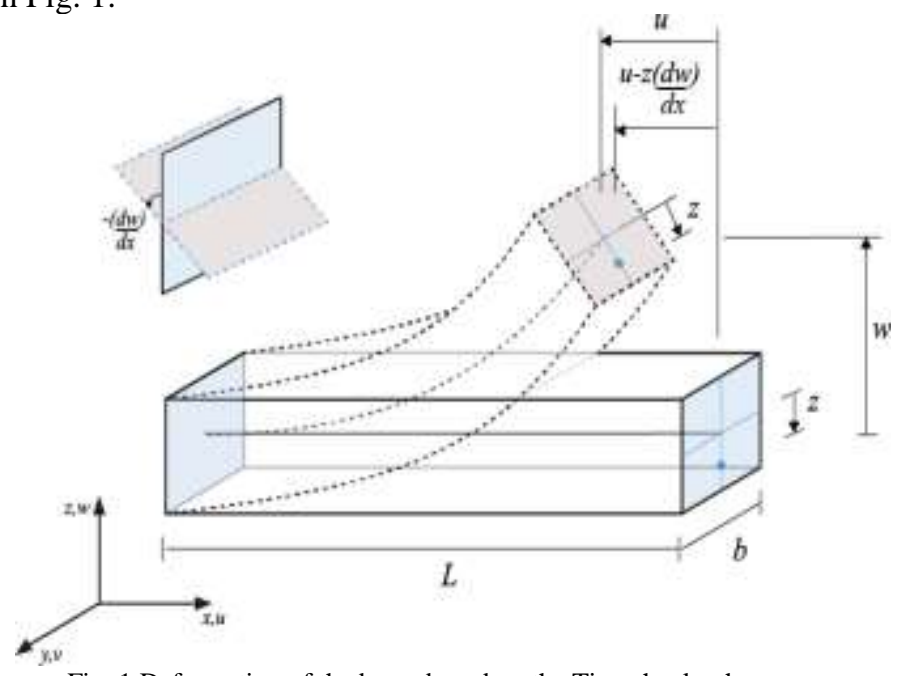

Fig. 1 Deformation of the beam based on the Timoshenko theory

The displacement field is represented by the following expression

$$
\begin{aligned}
& U_{1}=u(x)=u_{0}+z \phi_{1} \\
& U_{2}=v(x)=0 \\
& U_{3}=w(x)=w_{0}
\end{aligned}
$$

where the three degrees of freedom are showed: two displacements $u_{0}, w_{0}$ and a rotation $\phi_{1}$.

18th LACCEI International Multi-Conference for Engineering, Education, and Technology: "Engineering, Integration, and Alliances for a 
In Fig. 2, the geometric centroid ( $O$ point) is the origin of coordinates $(x, y, z)$ in the cross section of the element.

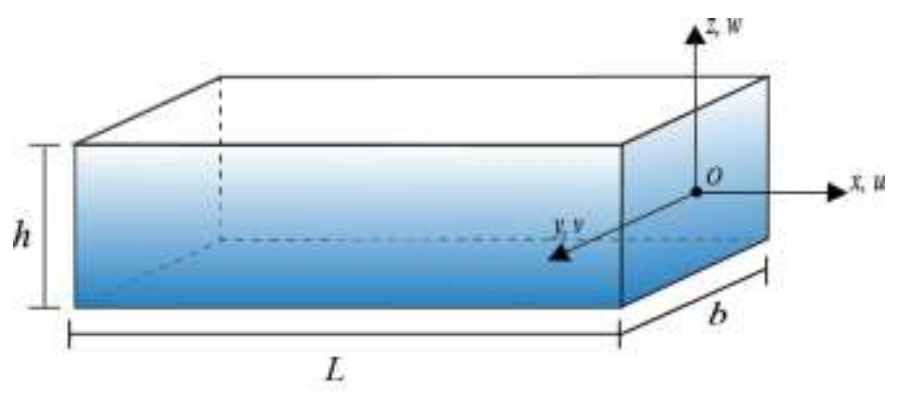

Fig. 2 Geometry and coordinates of the element

Kinematics equations are based on the Green-Lagrange strains tensor [10], namely

$$
\varepsilon_{i j}=(1 / 2)\left(U_{, x_{j}}^{i}+U_{, x_{i}}^{j}+U_{, x_{i}}^{k} U_{, x_{j}}^{k}\right)(i, j=1,2,3)
$$

where $U^{i}, U^{j}, U^{k}$ represent the displacement field showed in (1), and $x_{i}, x_{j}$ are the $x, y, z$ coordinates [11].

The complete strain tensor has nine terms. Six of them are independent due to symmetry $\varepsilon_{21}=\varepsilon_{12}, \varepsilon_{23}=\varepsilon_{32}, \varepsilon_{13}=\varepsilon_{31}$. Although, strains $\varepsilon_{22}=\varepsilon_{33}=\varepsilon_{12}=\varepsilon_{23}$ are equal to zero, because they are not deformations in that way. Hence, the components are

$$
\begin{aligned}
& \varepsilon_{11}^{0}=u_{, x}+(1 / 2)\left(w_{, x}\right)^{2} \\
& \varepsilon_{13}^{0}=(1 / 2)\left(\phi_{1}+w_{, x}\right) \\
& \varepsilon_{11}^{1}=\left(\phi_{1, x}\right)
\end{aligned}
$$

where super indexes 0 and 1 represent membrane strains and the bending strains, respectively.

The kinematics relations are expressed with Voigt notation. The governing equations of the structural system are given by

$$
\begin{array}{ll}
\delta u: & N_{1, x}=0 \\
\delta w: & Q_{1, x}+q+N_{1}\left(w_{, x^{2}}\right)=0 \\
\delta \varphi: & M_{1, x}-Q_{1}=0 .
\end{array}
$$

The stress resultants $N_{1}, Q_{1}, M_{1}$ are represented in the following equations

$$
\begin{aligned}
& N_{1}=\int_{-h / 2}^{h / 2} \sigma_{1}(1) d z \\
& M_{1}=\int_{-h / 2}^{h / 2} \sigma_{1}(z) d z \\
& Q_{1}=\int_{-h / 2}^{h / 2} \sigma_{5}(1) d z .
\end{aligned}
$$

\section{B. Functionally graded beams}

This two-phase functionally graded beam is composed of two materials: metal (at the bottom) and ceramic (at the top). The properties vary through the thickness of the element and are expressed in the following expression

$$
C_{i j k l}=C_{i j k l}^{c} f_{c}+C_{i j k l}^{m} f_{m}
$$

where the subscripts $c$ and $m$ are the expressions for ceramic and metal phases, respectively, and $f$ refers to the volume fractions which depend on the power-law denoted in the following formula

$$
f_{c}=[(z / h)+(1 / 2)]^{n} \quad f_{m}=1-f_{c}
$$

where $\mathrm{n}$ is the power-law index which varies from 0 (fully ceramic) to $\infty$ (fully metal).

The constitutive equations are expressed in (8) to get the relations between the stresses and the deformations

$$
\sigma_{i j}^{(r)}=C_{i j k l}^{(r+s)} \varepsilon_{k l}^{(s)} \text {. }
$$

The term $\sigma$ is the stress produced for the axial loads, shear loads and moment. Consequently, the fourth-order tensor $C$ refers to the integral along the element

$$
\begin{aligned}
C_{i j k l}^{(r)} & =\int_{-h / 2}^{h / 2} C_{i j k l}^{(r+s)} z^{(r)} d z \\
& =\left.C_{i j k l}^{(r+s)}\left(\left(z^{r+1}\right) /(r+1)\right)\right|_{-h / 2} ^{h / 2}
\end{aligned}
$$

for the next values:

$$
\begin{array}{lll}
(r=0, s=0) & C_{i j k l}^{(0)}=C_{i j k l}^{(0)}(h) & \\
(r=1, s=0) & C_{i j k l}^{(1)}=0 & (i=1,3) \\
(r=2, s=0) & C_{i j k l}^{(2)}=C_{i j k l}^{(2)}\left(h^{3} / 12\right) & (i=2) .
\end{array}
$$

Replacing (10) in (8), then in (5), the following expression is obtained

$$
\begin{aligned}
N_{1}^{(0)} & =C_{111}^{(0)} \varepsilon_{11}^{(0)}+C_{111}^{(1)} \varepsilon_{11}^{(1)}=A_{11} \varepsilon_{11}^{(0)}+B_{11} \varepsilon_{11}^{(1)} \\
M_{1}^{(1)} & =C_{1111}^{(1)} \varepsilon_{11}^{(0)}+C_{111}^{(2)} \varepsilon_{11}^{(1)}=B_{11} \varepsilon_{11}^{(0)}+D_{11} \varepsilon_{11}^{(1)} \\
Q_{1}^{(0)} & =A K S\left(C_{1313}^{(0)} \varepsilon_{13}^{(0)}\right)=A K S\left(A_{55} \varepsilon_{13}^{(0)}\right) .
\end{aligned}
$$

The term $Q_{1}$ is multiplied for a constant shear correction factor equal to $5 / 6$. Hence, the coefficients for the material stiffness are obtained from the following equation

$$
A_{i j}, B_{i j}, D_{i j}=\int_{-h / 2}^{h / 2}\left(\left(C_{i j}^{c}-C_{i j}^{m}\right)\left(f_{c}+C_{i j}^{m}\right)\right)\left(z-z_{c}\right)^{r} d z
$$

where $z_{c}$ is the position of the neutral axis when it changes due to the variation of the stresses as a consequence of the powerlaw index.

\section{Stability Analysis}

For the stability analysis, we utilize variational methods. For an equilibrium system, the total potential energy must be minimum. The displacement field is expressed through a fundamental as well as an incremental state [2]. Then

$$
U=\left\{\begin{array}{l}
u=u_{0}+u_{1} \\
w=w_{1}
\end{array}\right.
$$

where $u_{0}$ represent the displacement in a pure membrane state (fundamental state). Also, $u_{1}$ y $w_{1}$ are infinitesimally small increments (arbitrary perturbations).

Total potential energy can be represented with a Taylor's expansion

$$
\Delta \Pi=\Pi\left(U^{F}+U^{I}\right)-\Pi\left(U^{F}\right)=\sum_{n=1}^{4}(1 / n !) \delta^{n} \Pi .
$$


For the above expression the first variation $(\delta \Pi)$ must be equal zero, so the equation depends entirely on the second variation which must be nonnegative. Therefore, the critical buckling load is defined as the lowest load when the second variation of the potential energy is the minimum which is called Trefftz criterion [2]

$$
\delta\left[\delta^{2} \Pi\right]=0 .
$$

Replacing (15) in (3) and computing the second variation of the potential energy are expressed in the following expression

$$
\delta\left(\delta^{2} \Pi\right)=(1 / 2) \int_{\Omega}\left\{N_{1}^{0} w_{1, x} \delta w_{1, x}\right\} d x .
$$

\section{Finite Element Formulation}

The finite element model based on the Timoshenko beam theory is derived by the principle of virtual work. It allows the use of Lagrangian interpolation functions for the approximation of displacements and rotation.

The field variables are approximated by the following expression:

$$
\begin{gathered}
u=\sum_{j=1}^{m} U^{(j)} \Phi^{(j)}(x) \\
w=\sum_{j=1}^{m} W^{(j)} \Phi^{(j)}(x) \\
\phi_{1}=\sum_{j=1}^{m} \phi_{1}{ }^{(j)} \Phi^{(j)}(x) .
\end{gathered}
$$

Then, a matrix equation is obtained by substituting Equation (17) in (15), that means:

$$
[K-\lambda K g]=0 .
$$

In order to solve (18), an eigenvalue numerical method is utilized. Namely

$$
[K]\{\Delta\}=\lambda[K g]\{\Delta\}
$$

where $K$ and $K_{g}$ are the stiffness matrix and the geometric stiffness matrix, respectively; $\lambda$ is the eigenvalue. The critical buckling load is given in $\lambda$ and the buckling modes in $\Delta$.

\section{NUMERICAL RESULTS}

In this section, benchmark problems are studied to evaluate the accuracy of the present formulation for FGM Timoshenko beams.

\section{A. Isotropic beams}

It is necessary to clarify the difference between slenderness ratio $(L / R)$, which relate the length and radius of gyration, and slenderness relation $(L / H)$, which relate length and thickness of the beam, to get better understanding of the next topic.

The results obtained are compared with the Engesser formula [13] for isotropic beams. The Euler buckling theory does not take into consideration the effect of shear deformation. Therefore, for non-slender elements the formula is no longer valid. In order to consider this limitation, a clamped-clamped steel column with $E=200 \mathrm{GPa}$ and $v=0.3$ is evaluated for different slenderness ratios $(L / R)$. The critical buckling stress is normalized with the Young modulus to get a nondimensional value $\left(\sigma_{c r} / E\right)$. In Fig. 3, these three models (present, Engesser and Euler) are compared. The proposed formulation agrees with to Engesser formula. As we can see, the Euler theory over stiffens the element for the slenderness ratio minor of fifteen $(L / R<15)$, even exceeding its resistance limit.

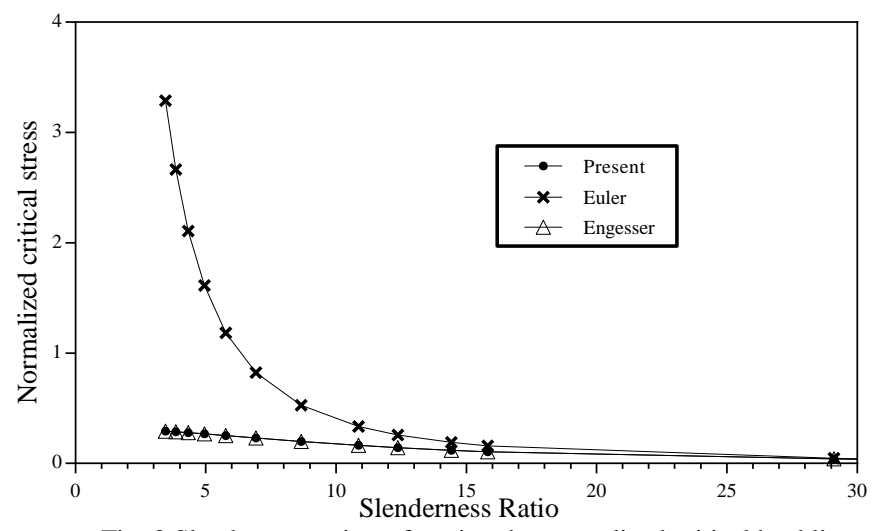

Fig. 3 Slenderness ratio as function the normalized critical buckling stress

To compare the results with other formulations in literature, the critical buckling load is normalized as in the following parameter:

$$
\bar{N}_{c r}=\left(N_{c r} b^{2}\right) /\left(E_{m} h^{3}\right) .
$$

To verify the precision of the method, an FGM column is evaluated. It is composed of alumina $\left(\mathrm{Al}_{2} \mathrm{O}_{3}\right)$ as the ceramic material and aluminum as metal with $E_{c}=380 \mathrm{GPa}$ and $E_{m}=$ $70 \mathrm{GPa}$, respectively. The Poisson's modulus $(v)$ is 0.23 and 0.3 for Li and Batra [6] and Kahya and Turan [7], respectively. Clamped-clamped, simple-simple and clamped-free are the boundary conditions for the analysis.

As shown in Table 1 and Table 2, the normalized critical buckling load is compared for two slenderness relations $(L / H)$ and nine power-law indexes $(n)$. A doubly clamped column is evaluated since this boundary condition requires of higher critical buckling load. The proposed formulation is compared with the method of Li and Batra [6] for doubly clamped beams.

\begin{tabular}{|c|c|c|c|}
\hline \multirow{2}{*}{$\begin{array}{c}\text { Slenderness } \\
\text { relation }(\mathrm{L} / \mathrm{H})\end{array}$} & \multirow{2}{*}{$\begin{array}{l}\text { Power-law } \\
\text { index (n) }\end{array}$} & \multicolumn{2}{|c|}{ Theory } \\
\hline & & Present & Li \& Batra [6] \\
\hline \multirow{9}{*}{5} & 0 & 154.352 & 154.350 \\
\hline & 0.5 & 103.224 & 103.220 \\
\hline & 1 & 80.498 & 80.498 \\
\hline & 2 & 62.615 & 62.614 \\
\hline & 5 & 50.384 & 50.384 \\
\hline & 7 & 47.332 & 47.332 \\
\hline & 10 & 44.267 & 44.267 \\
\hline & 100 & 31.231 & 31.231 \\
\hline & $\infty$ & 28.433 & 28.433 \\
\hline
\end{tabular}
The results show a close agreement with the analytical method of [6]. The critical load increases while the power-law index decreases. It represents that the buckling load depends on the material properties $\left(E_{c}>E_{m}\right)$. Also, the buckling load increases as soon as the slenderness relation raises.

TABLE 1

NORMALIZED CRITICAL LOAD FOR DOUBLY CLAMPED FGM BEAMS (L/H=5) 
TABLE 2

NORMALIZED CRITICAL LOAD FOR DOUBLY CLAMPED FGM BEAMS (L/H=10)

\begin{tabular}{cccc} 
Slenderness & \multirow{2}{*}{$\begin{array}{c}\text { Power-law } \\
\text { relation (L/H) }\end{array}$} & \multicolumn{2}{c}{ Theory } \\
\cline { 2 - 4 } & index (n) & Present & Li \& Batra [6] \\
\cline { 2 - 4 } & 0 & 195.343 & 195.340 \\
\cline { 2 - 4 } & 0.5 & 127.871 & 127.870 \\
\cline { 2 - 4 } 10 & 1 & 98.750 & 98.749 \\
\cline { 2 - 4 } & 2 & 76.981 & 76.980 \\
\cline { 2 - 4 } & 5 & 64.097 & 64.096 \\
\cline { 2 - 4 } & 10 & 61.062 & 61.062 \\
\cline { 2 - 4 } & 100 & 57.709 & 57.708 \\
\cline { 2 - 4 } & $\infty$ & 35.082 & 40.081 \\
\hline
\end{tabular}

In Fig. 4, an FGM beam is analyzed for three different boundary conditions (CC, SS and CF) with slenderness relation equal to five. Furthermore, the results are compared with the obtained on [7] and [14]. The buckling load obtained has an excellent approach to the benchmarking results. In other words, the proposed formulation match with the results of the authors and validates the present analysis.

To observe the influence of the slenderness relation and the power-law index, in Fig. 5 an FGM simple supported beam is evaluated. The critical buckling load increases as soon as the slenderness relation raises, but this increment is less than $1 \%$ when the slenderness relation $(L / H)$ is over 20. It happens for all power-law indexes cases because the material properties have more influence than the geometrical parameters.

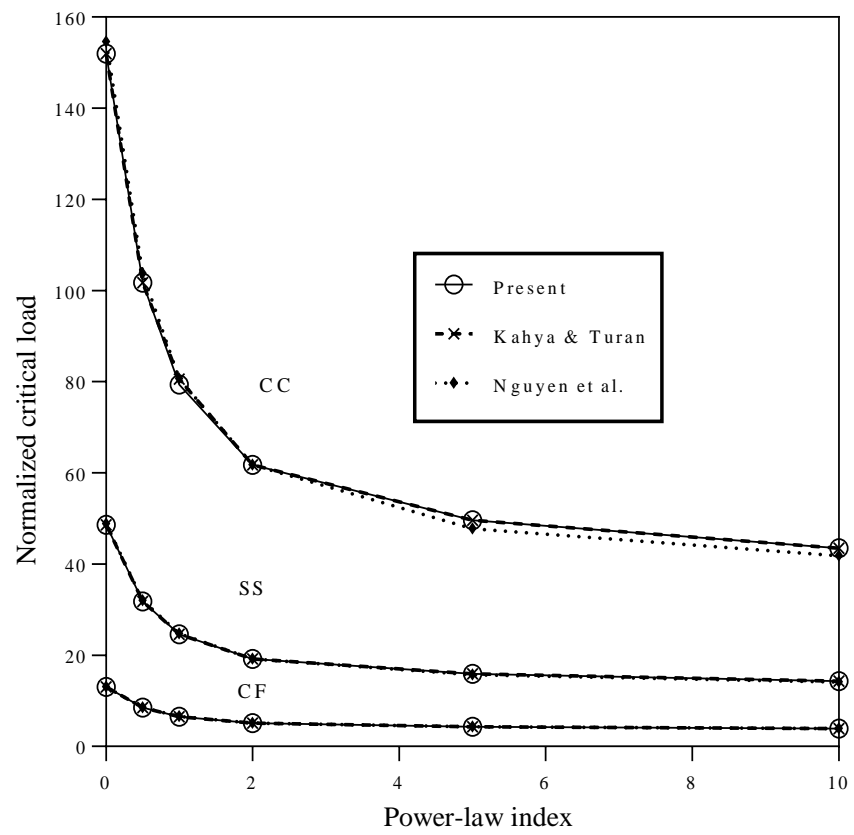

Fig. 4 FGM beams for different power law index

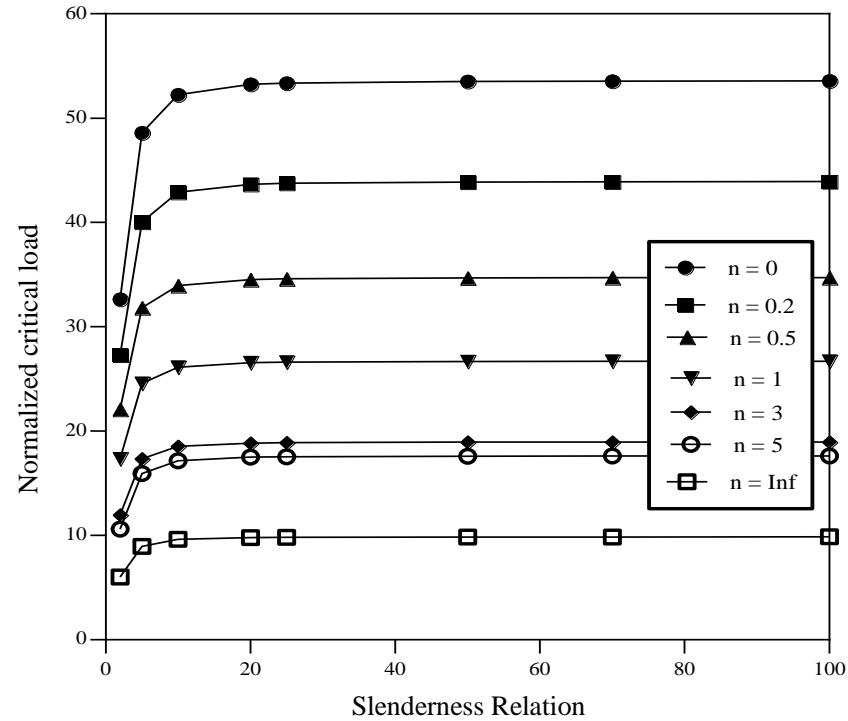

Fig. 5 Slenderness Relation under 8 different power-law indexes

\section{B. Buckling modes}

In this section numerical examples are presented to show the four principal buckling modes for different boundary conditions. Buckling modes are only relative displacements where the values vary among \pm 1 in the range displacements.

In Fig. 6-8, a clamped-free, simple supported and doubly clamped FGM column is evaluated. The slenderness ratio is $L / H=50$ and width $B=1$. The material is made of aluminum $\left(E_{m}=70 \mathrm{GPa}\right)$ and zirconia $\left(E_{c}=151 \mathrm{GPa}\right)$, Poisson modulus $\left(v_{m}=v_{c}=0.3\right)$ and the power-law index $(n=2)$. The example is computed with polynomials of fourth order $(P=4)$ and with a finite element mesh of four elements. Also, the first mode (on the left) represents the movement of the column for the critical buckling load, while the three other modes represent the most representative ones.

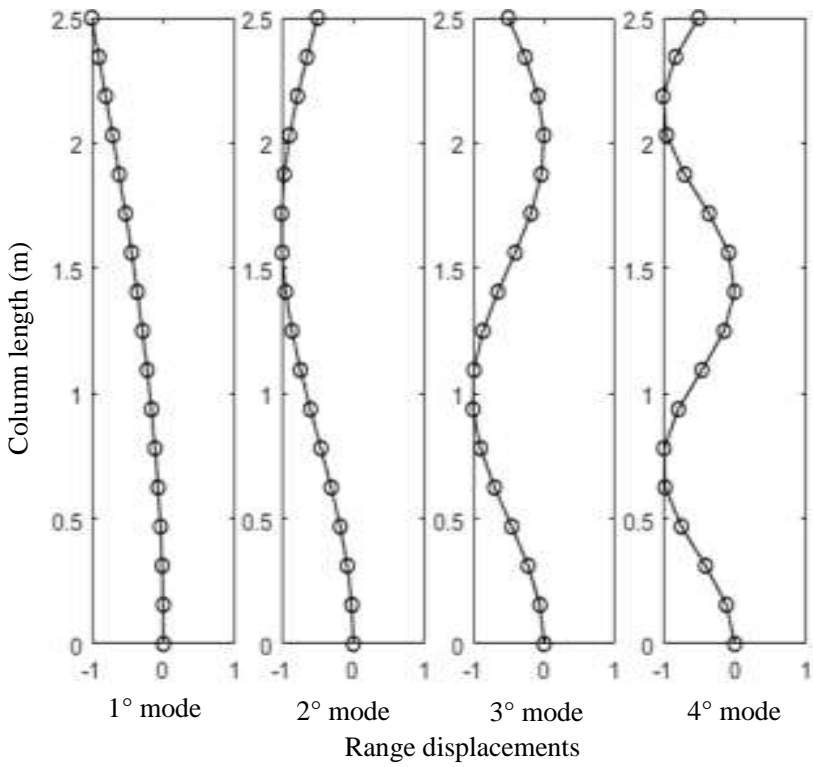

Fig. 6 Buckling mode shapes for a Clamped free column 

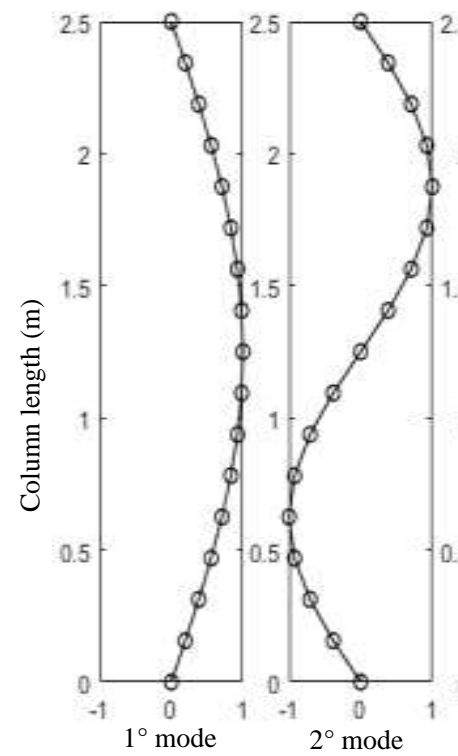

Range displacements

Fig. 7 Buckling mode shapes for a simple supported column

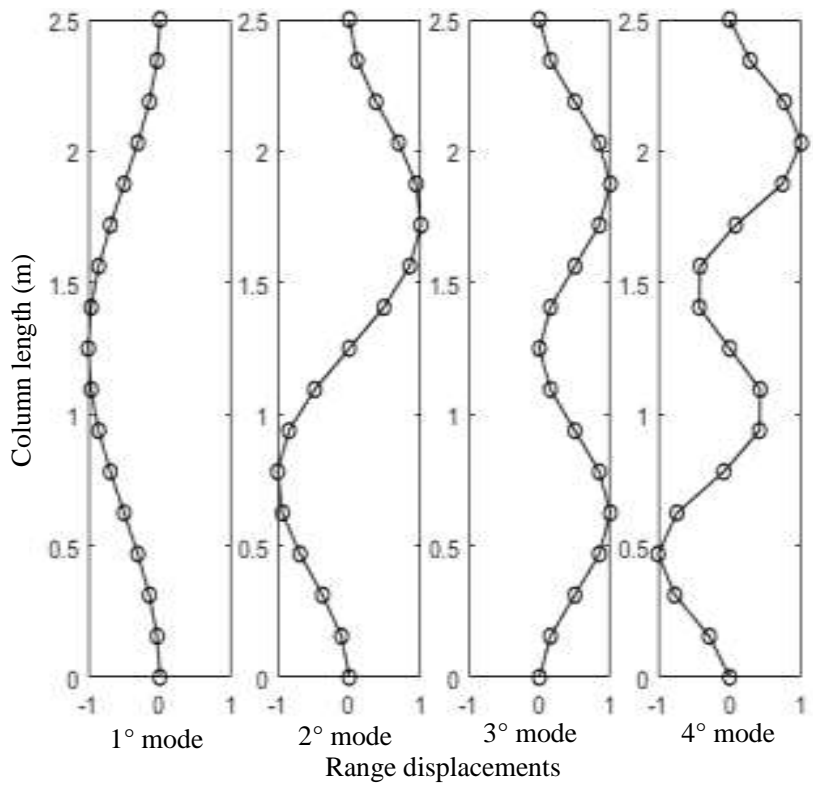

Fig. 8 Buckling mode shapes for a doubly clamped column

\section{CONCLUSION}

In the present study, a formulation to compute the critical buckling loading for functionally graded beams has been presented. The Timoshenko beam theory was used to develop a finite element model based on the principle of virtual work. The Trefftz criterion was utilized to derive the stability analysis. The formulation obtained high accuracy respect to the Engesser formula for isotropic cases. When comparing with the Euler formula, results varied significantly for ratios $L / R<15$. The classical formula does not take into consideration the shear deformation. The critical buckling load decreased when the index $n$ and the slenderness ratio $(L / H)$ decreased its values for functionally graded beams.

\section{REFERENCES}

[1] R. Jones, "Buckling of Bars,Plates, and Shells." 2006.

[2] D. Brush and B. Almroth, Buckling of Bars, Plates and Shells. McGraw-Hill, 1975.

[3] T. Hirano, T. Yamada, J. Teraki, M. Niino, and A. Kumakawa, “A study on a functionally gradient material design system for a thrust chamber," Int. Symp. a Sp. Technol. Sci., vol. 16 th, pp. 375-380, 1988.

[4] K. Soncco, X. Jorge, and R. Arciniega, "Postbuckling Analysis of Functionally Graded Beams," IOP Conf. Ser. Mater. Sci. Eng., vol. 473, p. 012028, Feb. 2019.

[5] J. N. Reddy and R. A. Arciniega, "Mechanical and Thermal Buckling of Functionally Graded Ceramic-Metal Plates," vol. 1, no. 1, pp. 1-21, 2004.

[6] S. R. Li and R. C. Batra, "Relations between buckling loads of functionally graded timoshenko and homogeneous euler-bernoulli beams," Compos. Struct., vol. 95, pp. 5-9, 2013.

[7] V. Kahya and M. Turan, "Finite element model for vibration and buckling of functionally graded beams based on the first-order shear deformation theory," Compos. Part B Eng., vol. 109, pp. 108-115, 2017.

[8] Y. Huang and X. F. Li, "Buckling of functionally graded circular columns including shear deformation," Mater. Des., vol. 31, no. 7, pp. 3159-3166, 2010.

[9] T. P. Vo, H. T. Thai, T. K. Nguyen, A. Maheri, and J. Lee, "Finite element model for vibration and buckling of functionally graded sandwich beams based on a refined shear deformation theory," Eng. Struct., vol. 64, pp. 12-22, 2014.

[10] P. GN and R. JN, "Nonlinear transient thermoelastic analysis of functionally graded ramic-metal plates," Int. J. Solids Struct., vol. 35, no. 1987, pp. 4457-71, 1998.

[11] K. Åhlander, "Einstein summation for multidimensional arrays," Comput. Math. with Appl., vol. 44, no. 8-9, pp. 1007-1017, 2002.

[12] M. R. (Mohamad R. Eslami, Buckling and postbuckling of beams, plates, and shells. 2018.

[13] Z. P. ; Bazant and L. Cedolin, Stability of structures, $2^{\circ}$. Donver Publications, Inc, 2003.

[14] T. Nguyen, T. T. Nguyen, T. P. Vo, and H. Thai, "Vibration and buckling analysis of functionally graded sandwich beams by a new higher-order shear deformation theory," Compos. Part B, 2015. 